\title{
Elimination of the Chirp of Optical Pulses through Cascaded Nonlinearities in Periodically Poled Lithium Niobate Waveguides
}

\author{
Kwang Jo Lee ${ }^{(1)}$, Sheng Liu ${ }^{(1)}$, Katia Gallo ${ }^{(2)}$, Periklis Petropoulos ${ }^{(1)}$, and David J. Richardson ${ }^{(1)}$ \\ (1) Optoelectronics Research Centre, University of Southampton, Southampton, SO17 1BJ, United \\ Kingdom, kjl@orc.soton.ac.uk \\ (2) Department of Applied Physics, Royal Institute of Technology (KTH), 10691 Stockholm, Sweden
}

\begin{abstract}
We propose and demonstrate a novel method for the elimination of arbitrary frequency chirp from short optical pulses. The technique is based on the combination of two cascaded second-order nonlinearities in two individual periodically poled lithium niobate waveguides.
\end{abstract}

\section{Introduction}

Picosecond-long optical pulses are widely used in diverse applications, such as optical time division-multiplexing communications, optical sensors, and optical imaging systems. Such short optical pulses can be generated using either mode-locked lasers, gain-switched laser diodes, or even externally modulated continuous-wave (CW) light sources. However, the generated optical pulses often have some intrinsic frequency chirps and, especially in transmission systems, the cumulative effects of various optical nonlinearities can either intensify this chirp or induce additional nonlinearity in the transmitted optical pulses.

A certain length of a dispersion compensating fibre (DCF) is usually employed to remove the linear chirp induced in the optical pulses. In recent years, several reliable techniques to generate chirp-free pulses have also been reported, which are based on either electro-optic phase modulation followed by nonlinear soliton compression $^{1}$, or the use of polarization and zero-chirp intensity modulations ${ }^{2}$.

However, the afore-mentioned techniques require knowledge of the exact chirp characteristics, and the related system parameters for the elimination of the chirp should be designed accordingly in each case. This introduces complexity in practical applications, and therefore, makes these approaches less attractive.

In recent years, the use of cascaded secondorder nonlinear processes in periodically poled lithium niobate (PPLN) waveguides has attracted considerable interest as a promising route to realise all-optical signal processing ${ }^{3}$. The technology provides for high nonlinear coefficients, ultra-fast optical response, bit rate and modulation format transparency, low cross talk, no spontaneous emission noise, and no intrinsic frequency chirp. Cascaded secondharmonic and difference-frequency generation (cSHG/DFG) and cascaded sum- and difference -frequency generation (cSFG/DFG) have both been exploited in various all-optical signalprocessing applications, such as wavelength conversion $^{4}$, format conversion ${ }^{5}$, logic gates ${ }^{6}$, tunable optical time delays ${ }^{7}$, and phase sensitive amplification $^{8}$.

In this paper, we propose and demonstrate an effective method to remove intrinsic frequency chirps induced in ps-optical pulses. The technique relies on the generation of a conjugated replica of the input chirped pulse in a first PPLN waveguide via CSHG/DFG, followed by the nonlinear interaction of the two phseconjugated signals via cSFG/DFG in a second PPLN waveguide for the generation of the chirpfree pulse. Our new scheme has a distinguished advantage over the techniques previously reported in that even unknown or nonlinear chirps can be erased, because it relies on the pulse itself and its conjugated replica to interact with each other in order to cancel out their chirps.

\section{Principles of operation}

Figure 1 illustrates our scheme for the elimination of chirp in pulses based on the combination of cSHG/DFG and cSFG/DFG in two cascaded PPLN waveguides. The second harmonic (SH) wave of an input CW pump is first generated in the first PPLN device, which is

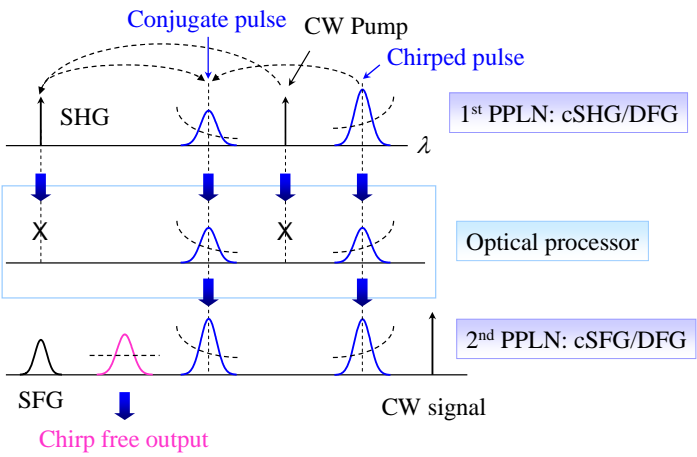

Fig. 1: Illustration of chirp elimination in the short pulse based on both cSHG/DFG and cSFG/DFG in two cascaded PPLN waveguides. 
accompanied by DFG between this $\mathrm{SH}$ signal and the initially chirped input pulse to produce the phase-conjugate counterpart with opposite chirp. All of the resulting optical waves after the first PPLN are subsequently passed through an optical processor. This is an amplitude filter which rejects both the pump and the SH waves, and equalises the optical power of the input pulse with that of its conjugate. Now the two pulses after the optical processor interact with each other as two pulsed pumps to generate the sum-frequency (SF) wave in the second PPLN waveguide. The SF optical field is expressed by the product of the two original optical fields, which means that the imaginary part in the SF field is cancelled out and thus any frequency chirp is erased. The SF pulse then interacts with a second CW beam, and which finally generates the chirp-free output pulse as illustrated in Fig. 1.

\section{Experimental results}

Figure 2 shows the experimental setup used to realise our chirp-free pulse generation system. Two 30-mm-long fibre pigtailed PPLN waveguides (HC Photonics Corp.) were used to instigate the two kinds of second-order nonlinear processes, and their SHG phase matching wavelength was $1546 \mathrm{~nm}$ at $50^{\circ} \mathrm{C}$ and $42^{\circ} \mathrm{C}$, respectively. A CW laser operating at $1546.0 \mathrm{~nm}$ was used as the pump source for the first PPLN device. A 10-GHz, 2-ps pulses generated from a mode locked erbium glass oscillator (ERGO) at $1552 \mathrm{~nm}$ were launched into 150-m of single mode fibre (SMF) to generate the input chirped pulses. The CW pump and the chirped pulses were combined in a 3-dB coupler and then amplified before being launched into the first PPLN waveguide. The total power at the input of the waveguide was restricted to $21 \mathrm{dBm}$ to protect its coupling connectors (note that the same condition was applied to the second PPLN waveguide as well). As discussed in Fig. 1, the $\mathrm{SH}$ wave is generated from the CW pump in the PPLN waveguide, which then interacts with the chirped pulse via the DFG process to produce the conjugate replica. Figure 3(a) shows the measured spectrum of the signal at the output of the first PPLN waveguide. The optical signal to noise ratio (OSNR) of the CSHG/DFG signal was measured to $12.2 \mathrm{~dB}$. The optical processor (Finisar Wave Shaper 4000E) was used to filter out both the pump and the $\mathrm{SH}$ waves, and to equalise the optical power of the input chirped pulse with that of conjugate pulse as shown in Fig. 3(b). Power equalisation of the two conjugate signals is desirable, in order to maximise the efficiency of the nonlinear processes in the second PPLN waveguide. The two signals were then amplified and combined with a 1558-nm CW light in a second 3-dB coupler. All three waves were launched into the

Pulsed signal @1552nm

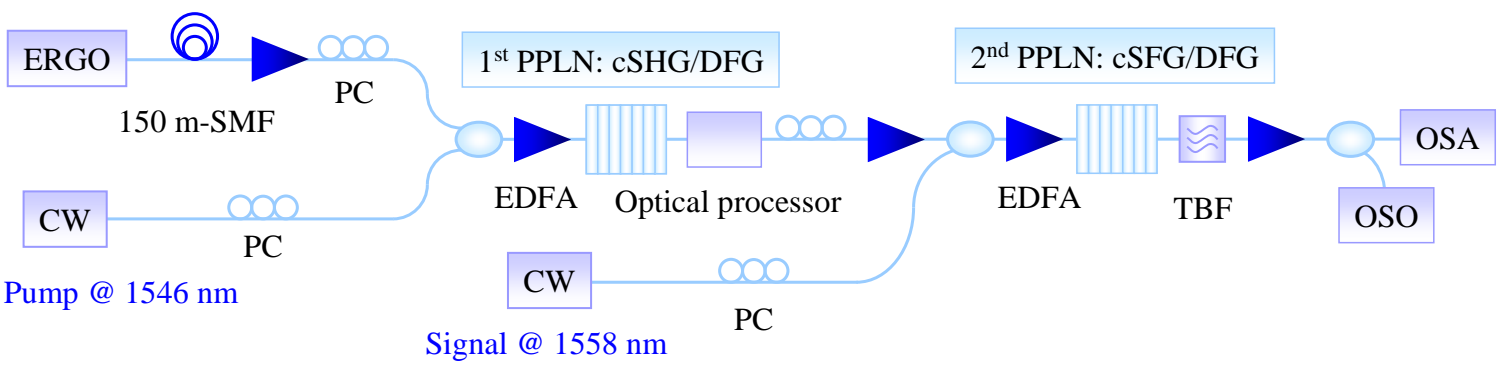

Fig. 2: Experimental setup used to generate the chirp-free pulse via a combination of cSHG/DFG and cSFG/DFG. PC: polarization controller, EDFA: erbium-doped fibre amplifier, TBF: tunable bandpass filter, OSO: optical sampling oscilloscope, OSA: optical spectrum analyser.
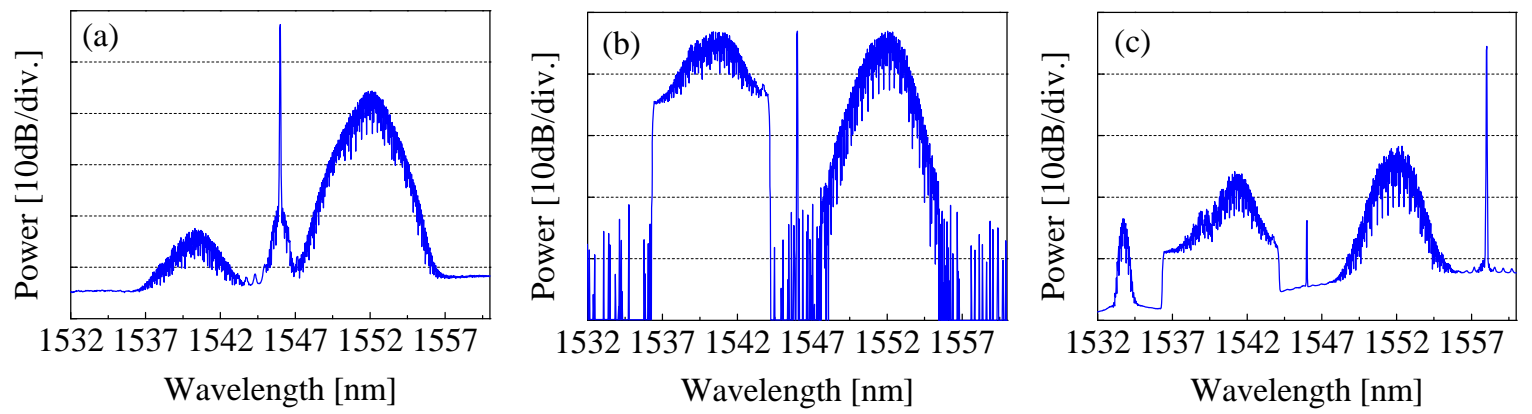

Fig. 3: Spectral traces measured after (a) the first PPLN waveguide, (b) the optical processor, and (c) the second PPLN waveguide. 

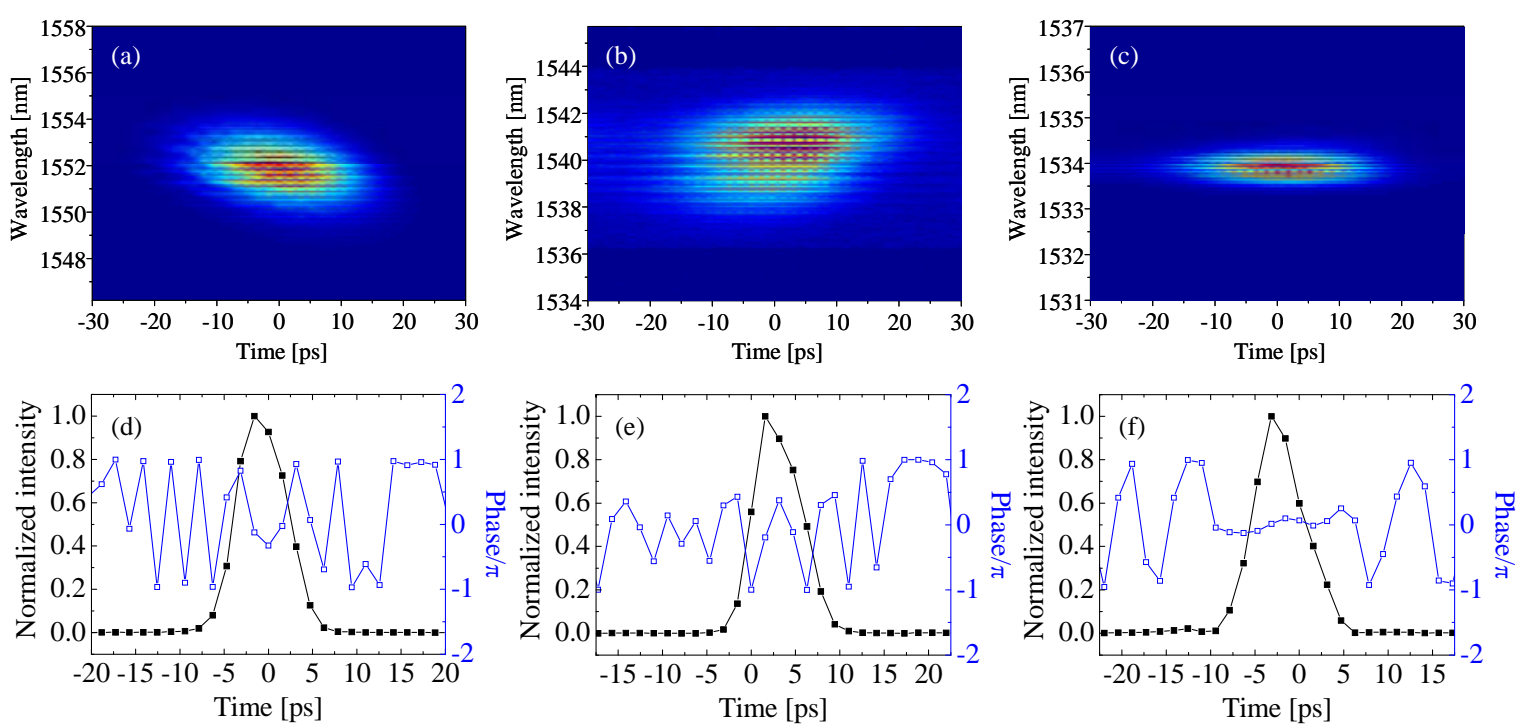

Fig. 4: Measured FROG traces for (a) the input chirped pulse, (b) its conjugated replica, and (c) the resultant chirp-free output pulses. Retrieved normalized intensities and phase profiles of (d) the input chirped pulse,

(e) its conjugated replica, and (f) the resultant chirp-free output pulse.

second PPLN waveguide to interact with each other via cSFG/DFG as described in Fig. 1. The resultant spectrum, measured after the second PPLN device, is shown in Fig. 3(c), and the measured OSNR of the cSFG/DFG output signal at $1533.7 \mathrm{~nm}$ was $14 \mathrm{~dB}$.

In order to assess the performance of the system and its capacity to eliminate the chirp of optical pulses, we performed linear frequency resolved optical gating (I-FROG) measurements, using an electro-optic modulator as the sampling gate ${ }^{9}$. Figure $4(a)-(c)$ shows the spectrograms obtained for the input chirped pulse, its conjugated replica, and the resultant chirp-free output pulse respectively. Normalized intensities and phases of the corresponding retrieved pulses are also shown in Fig. 4(d)-(f). A filter, tunable both in bandwidth and centre wavelength (Alnair Labs.), was used after the second PPLN waveguide to extract and characterise each pulse separately. As shown in Fig. 4(a, d) and $(b, e)$, the chirp of the conjugated pulses is opposite to that of the original signal, whereas the chirp in the cSFG/DFG signal has been cancelled out to produce a chirp-free output, as can be seen in Fig. 4(c) and (f). Additional measurements with an optical sampling oscilloscope (EXFO PSO100) and an optical spectrum analyser confirmed pulse widths of $6.5 \mathrm{ps}$ and $6.6 \mathrm{ps}$ for the input and output pulses respectively, and a time-bandwidth product for the chirp-free output pulses of 0.48 , which indicating a good quality close to the transform-limited pulses.

\section{Conclusion}

We have demonstrated an effective method to erase the chirp of ps-long pulses and generate a chirp-free output. The technique, which is based on a combination of cascaded nonlinear effects in two different PPLN waveguides, operates independently of the chirp characteristics and does not require any prior knowledge of the chirp.

\section{Ackowledgement}

The research leading to these results has received funding from the UK EPSRC under grant agreement EP/F032218/1. Katia Gallo gratefully acknowledges support from the EU under grant agreement PIEF-GA-2009-234798.

\section{References}

1. T. Otsuji et al., IEEE J. Sel. Top. Quantum Electron. 2, 643 (1996).

2. S. Pan et al., Opt. Lett. 15, 2186 (2009).

3. C. Langrock et al., J. Lightwave Technol. 24, 2579 (2006).

4. K. Gallo et al., Appl. Phys. Lett. 71, 1020 (1997).

5. K. J. Lee et al., Opt. Express 18, Accepted (2010).

6. J. E. McGeehan et al., Electron. Lett. 43, 409 (2007).

7. Y. Wang et al., IEEE Photon. Technol. Lett. 19, 861 (2007).

8. K. J. Lee et al., Opt. Express 17, 20393 (2009).

9. K. T. Vu et al., IEEE Photon. Technol. Lett. 20, 505 (2008). 